\section{Prevalência e fatores associados às geo-helmintíases em crianças residentes em municípios com baixo IDH no Norte e Nordeste brasileiros}

\author{
Prevalence and factors associated with \\ geohelminth infections in children living in \\ municipalities with low HDI in North and \\ Northeast Brazil
}

\author{
Eduardo Oyama Lins Fonseca 1 \\ Maria Glória Teixeira ${ }^{2}$ \\ Maurício L. Barreto 2 \\ Eduardo Hage Carmo 3 \\ Maria da Conceição N. Costa ${ }^{2}$
}

\footnotetext{
${ }^{1}$ Laboratório Central de Saúde Pública Prof. Gonçalo Moniz, Salvador, Brasil. 2 Instituto de Saúde Coletiva Universidade Federal da Bahia, Salvador, Brasil. 3 Secretaria de Vigilância da Saúde, Ministério da Saúde, Brasília, Brasil.

Correspondência M. G. Teixeira Instituto de Saúde Coletiva Universidade Federal da Bahia.

Rua Pancararé 221 Salvador, $B A$ 41650-640, Brasil. magloria@ufba.br
}

\begin{abstract}
Stool surveys were conducted to estimate the prevalence and identify risk factors for geohelminth infections among children in ten Brazilian municipalities with low human development indices (HDI). Socioeconomic and environmental data were obtained from the children's parents or guardians, and stool samples were examined. The proportion of geohelminth infections according to target variables was calculated. Risk factors were evaluated using multilevel logistic regres sion. Of the 2,523 children, 36.5\% were infected with one or more geohelminths (Ascaris lumbricoides, 25.1\%; hookworm, 15.3\%; Trichuris trichiura, 12.2\%). Overall prevalence of geohelminth infections was $45.7 \%$ in rural areas and $32.2 \%$ in urban areas. Low family income $(O R=1.75$; 1.38 2.23), low maternal schooling $(O R=1.69 ; 1.39$ 2.06), presence of garbage near the home $(O R=$ $1.50 ; 1.22-1.84$ ), and number of individuals in the household $(O R=1.41 ; 1.17-1.71)$ were associated with infection. In conclusion, geohelminth infections were closely related to socioeconomic conditions, thus emphasizing the importance of targeted public interventions to improve living conditions as part of sustainable prevention.
\end{abstract}

Helminthiasis; Parasitic Diseases; Child

\section{Introdução}

A elevada magnitude e ampla distribuição geográfica das enteroparasitoses, aliadas às repercussões negativas que podem causar no organismo humano, têm conferido a essas infecções uma posição relevante entre os principais problemas de saúde da população.

Especialmente em países em desenvolvimento que não alcançaram êxito no seu controle, as parasitoses intestinais se mantêm como importante causa de morbidade 1 , chegando a atingir índices de até $90 \%$ nos estratos populacionais de níveis sócio-econômicos mais baixos 2. Embora, per $s i$, as enteroparasitoses não constituam risco imediato de morte na infância, a sua relação com a diarréia e a desnutrição pode colocar em risco a sobrevivência ${ }^{3}$ e o adequado desenvolvimento físico e mental da criança 4 .

As infecções produzidas por enteroparasitos estão presentes, praticamente, em todas as zonas tropicais e subtropicais do planeta 5 . Estima-se que, atualmente, mais de um bilhão de indivíduos em todo mundo albergam pelo menos uma espécie de parasita intestinal, sendo Ascaris lumbricoides, Trichuris trichiura e ancilostomídeos os que apresentam freqüências mais elevadas 6 .

Estima-se que cerca de $20 \%$ a $30 \%$ da população da América Latina esteja infectada por geohelmintos 7. Porém, a distribuição das parasitoses varia entre países e entre áreas dentro de um mesmo país. Enquanto a prevalência para 
A. lumbricoides e T. trichiura, entre crianças em idade pré-escolar e escolar, em área periférica de Buenos Aires, Argentina, em 2005 foi de 19,2\% 8, no Município de Armenia, Colômbia, a prevalência para A. lumbricoides e T. trichiura em préescolares, neste mesmo ano, foi de $2,4 \%$ e $2,1 \%$, respectivamente 9 .

Exemplo de variação intrapaís pode ser observado no Brasil, onde a maioria dos estudos sobre a prevalência de parasitos intestinais, geralmente, reflete apenas a realidade de pequenas localidades, ou seja, não representa a situação do país como um todo. Assim, no ano de 2005, em área urbana do Município de Santa Izabel, Amazonas, localizada na Região Norte do país, encontrou-se prevalência de aproximadamente $40 \%$ para A. lumbricoides, $24 \%$ para T. trichiura $\mathrm{e}$ $5 \%$ para ancilostomídeos, em crianças com idades entre 6 meses e 7 anos 10. Já no Sudeste do país, entre 2000 e 2002 no Município de Estiva Gerbi, São Paulo, a positividade era de apenas 1,5\% para A. lumbricoides, 0,1\% para T. trichiura e $0,1 \%$ para ancilostomídeos, entre crianças na faixa etária de 0 a 7 anos residentes em áreas urbana e rural 11. Vale salientar que é esta última região que concentra a maioria dos estudos sobre esse tema.

Sabe-se que as acentuadas desigualdades sócio-econômicas observadas no Brasil, aliadas ao desordenado processo de urbanização, levam parcelas da população a viverem em precárias condições de vida. A ocupação de áreas periféricas das cidades nas quais as condições de saneamento são deficitárias torna a qualidade de vida não muito diferente da área rural ou, muitas vezes, em piores condições 12 propiciando a transmissão das parasitoses intestinais 13,14.

Por conta da multiplicidade de fatores envolvidos na sua ocorrência, muitos dos quais de difícil equacionamento pelo setor saúde neste país, tais parasitoses persistem como um importante problema de saúde na população brasileira, apesar dos conhecimentos científicos e avanços tecnológicos disponíveis para tratamento e prevenção. A propósito, este pode ser um dos fatores que contribuíram para a idéia equivocada de que as enteroparasitoses deixaram de constituir um problema de saúde pública em todo o mundo.

Todavia, por reconhecer que essas infecções continuam prevalecendo no país, o governo brasileiro lançou, em 2005, o Plano Nacional de Vigilância e Controle das Enteroparasitoses 15 e, em 2007, o Programa de Aceleração do Crescimento (PAC) que prevê ações em saneamento básico, tendo como meta a redução de doenças infecciosas e parasitárias, entre as quais estão incluídas as parasitoses intestinais.
Em vista das iniciativas, este estudo teve como objetivo descrever a ocorrência de geohelmintíases em crianças residentes em municípios do Norte-Nordeste brasileiro e identificar fatores de risco ambiental e sócio-econômicos relacionados com a sua distribuição, tendo como propósito produzir informações que possam subsidiar o planejamento, programação e avaliação de intervenções voltadas para a prevenção e controle das referidas infecções.

\section{Material e métodos}

Foi realizado um inquérito transversal, entre março a setembro de 2005, em 10 dos 22 municípios brasileiros incluídos no Projeto de Avaliação de Impacto na Saúde das Ações de Saneamento 16, do qual este se constitui num subprojeto. No ano 2000, a Fundação Nacional de Saúde (FUNASA) instou os municípios brasileiros cujo Índice de Desenvolvimento Humano (IDH), em 1991, foi $<0,500$ e que atendessem aos critérios da Portaria $n^{o} .176$ de 28 de março de 2000 17, a apresentar projetos para captar recursos destinados à execução de obras de saneamento (abastecimento de água, melhorias sanitárias domiciliares e esgotamento sanitário). Entre janeiro de 2001 até julho de 2002, cerca de 1.846 desses municípios firmaram convênio com a FUNASA. Mediante tal cadastro, uma amostra intencional de 22 municípios foi selecionada considerando-se diferentes portes populacionais e maiores valores de recursos solicitados, visando privilegiar aqueles que iriam ser objeto de intervenções de saneamento mais amplas 16. A disponibilidade de informação sobre todas as variáveis sócio-ambientais necessárias para o estudo foi o critério adotado para a seleção dos dez municípios. Dentre eles, seis pertenciam à Região Nordeste (Independência e Mucambo no Ceará, São José do Peixe e Ribeiro Gonçalves no Piauí e Santa Helena e Maracaçumé no Maranhão) e quatro à Região Norte (Bragança e Ourém no Pará e Dianópolis e Itacajá no Tocantins).

O tamanho da amostra foi determinado com o auxílio do programa Epi Info 6.04d (Centers for Disease Control and Prevention, Atlanta, Estados Unidos), com base na literatura específica considerando-se como $20 \%$ a freqüência esperada do evento, $10 \%$ a pior freqüência aceitável e intervalo de $95 \%$ de confiança, aplicando-se a técnica de cálculo para populações finitas, para cada município. Em nenhum dos municípios estudados, o tamanho da amostra ultrapassou 60 crianças. Entretanto, para aumentar o poder do estudo a fim de permitir a estratificação das variáveis a serem analisadas, decidiu-se aplicar 
efeito desenho igual a 5, o que ampliou a amostra para 300, sempre que possível.

Tendo em vista que todas as famílias eram cadastradas no Programa de Agentes Comunitários de Saúde (PACS), inicialmente, foram numeradas as fichas de inscrição das famílias e, para cada um dos municípios realizou-se o sorteio aleatório simples daquelas a serem incluídas no estudo. Se na família havia somente uma criança da faixa etária de interesse, era considerada para o estudo. Se havia duas, uma delas era selecionada por sorteio; quando havia três ou mais crianças, apenas duas delas eram sorteadas. Caso não fossem encontradas crianças elegíveis em uma família sorteada, sorteavam-se tantas famílias quantas fossem necessárias até encontrar uma que, pelo menos, estivesse na faixa etária estabelecida. Ao final, participaram do estudo 2.523 crianças da referida faixa etária (292 em Dianópolis e em Itacajá, 288 em Ourém, 272 em Independência e em Santa Helena; 261 em Bragança, 258 em São José do Peixe, 231 em Maracaçumé e 102 em Mucambo).

Um questionário estruturado foi aplicado à mãe ou responsável com intuito de obter informações sobre condições físicas do domicilio, abastecimento de água, presença de lixo no peridomicílio (distância de até $30 \mathrm{~m}$ da casa), zona de residência, renda familiar, escolaridade materna, idade e sexo da criança e se a mesma realizou exame de fezes e/ou usou anti-helmínticos nos últimos seis meses. Nesta última situação, a criança não era incluída no estudo. Após a entrevista, foram doados recipientes para a coleta de material fecal da criança (uma única coleta por criança). No dia seguinte, os coletores eram recolhidos pelos agentes de saúde do PACS e encaminhados ao laboratório do Centro de Saúde do município, onde um técnico do projeto preparava duas lâminas por amostra, e parte das fezes era colocada em formol a $10 \%$ para posterior exame. Cada amostra foi examinada concomitantemente pelos métodos de Kato modificado por Katz 18, e sedimentação espontânea 19. Em média, os exames eram realizados no Posto Avançado do Instituto de Saúde Coletiva (ISC) da Universidade Federal da Bahia (UFBA), em Salvador, duas semanas após a coleta. Os resultados dos exames e o medicamento específico para o tratamento das crianças foram encaminhados à Secretaria de Saúde de cada município.

Realizou-se a análise descritiva e calculouse a proporção de geo-helmintíases (total e para cada tipo de parasita) segundo estratos de cada variável (sexo, idade, zona de residência, escolaridade da mãe, renda da família, número de moradores na casa, presença de água encanada, lixo próximo de casa, número de cômodos na casa), sendo a diferença entre elas verificada pela razão de proporções, aceitando-se nível de significância de 0,05 .

A existência de associação entre os possíveis fatores de risco e ocorrência de geo-helmintíases foi avaliada, inicialmente, mediante regressão logística bivariada. Pelo fato de na literatura todas as variáveis investigadas serem consideradas como importante fator de risco para a ocorrência de enteroparasitoses, mesmo aquelas que nesta análise não apresentaram significância estatística, todas elas foram incluídas em modelo multivariado, utilizando-se a técnica backward. Esta modelagem foi realizada por meio de análise multinível 20 (ou de efeitos aleatórios) de modo a assegurar que a variância não seria subestimada, visto que esse método considera o efeito cluster que pode ocorrer quando os dados são obtidos por conglomerados (no presente estudo, definidos pelos municípios). O referido efeito diz respeito à possibilidade de respostas mais similares, no que se refere a confundidores, entre indivíduos pertencentes ao mesmo conglomerado, do que entre aqueles que pertencem a conglomerados distintos. O programa Stata 7.0 (Stata Corp., College Station, Estados Unidos) foi utilizado para análise dos dados.

Esta investigação foi aprovada pelo Comitê de Ética do ISC/UFBA (protocolo nº 011-04/ CEP-ISC).

\section{Resultados}

Das 2.523 crianças incluídas no estudo, 52\% eram do sexo masculino, 60,9\% tinham entre 10 e 14 anos de idade e $67,6 \%$ residiam na área urbana. A maioria delas $(61,4 \%)$ tinha mães que possuíam mais de 3 anos de estudo, entretanto apenas 5,4\% das mães tinham 8 ou mais anos de escolaridade. Mais de $90 \%$ das crianças pertenciam a famílias com renda mensal até dois salários mínimos e para quase $75 \%$ delas a renda mensal era igual ou inferior a um salário mínimo (R \$300,00 à época do inquérito); 53,1\% residiam em domicílios com até 5 moradores, 78,4\% em domicílios sem água encanada, $53,5 \%$ em moradias com lixo na sua proximidade e, perto de $60 \%$ residiam em casa com 4 ou menos cômodos. Foi de 8,9 $\pm 2,4$ anos o valor médio e o desvio-padrão para a idade das crianças estudadas; 3,2 $\pm 1,5$ anos para a escolaridade das mães; $2,1 \pm 1,3$ salários para a renda familiar mensal e; $2,3 \pm 0,7$ para o número de pessoas no domicílio. No que se refere à distribuição das crianças segundo procedência (urbana/rural), constata-se diferença estatisticamente significante para escolaridade da mãe, renda familiar, presença de lixo próximo de casa, água encanada 
no domicílio e número de pessoas na residência (Tabela 1).

A proporção de geo-helmintíase para o total de crianças examinadas foi de $36,5 \%$. A espécie mais freqüente foi $A$. lumbricoides (25,1\%), seguida pelos ancilostomídeos (12,2\%) e T. trichiura $(12,2 \%)$. Os maiores índices foram encontrados em Santa Helena (64,7\%), Ourém (63,5\%) e Maracaçumé (61,5\%). Os municípios de Independência e Mucambo e Itacajá apresentaram os menores índices, com valores de 12,5\%, 13,7\% e 17,1\%, respectivamente.

Crianças que viviam em casas com lixo nas proximidades (48\%), cujas mães tinham escolaridade igual ou inferior a 3 anos de estudo $(46,9 \%)$, residentes em domicílios com renda familiar igual ou inferior a 1 salário mínimo $(41,4 \%)$, com 4 ou menos cômodos (41\%) e com mais de 5 pessoas $(44,8 \%)$ apresentaram freqüência maior de geo-helmintíases (Tabela 2).
Padrão de distribuição semelhante foi observado para cada uma das espécies de geohelmintos analisadas. O sexo masculino apresentou maior prevalência, porém com diferença estatisticamente significante apenas para ancilostomídeos. A. lumbricoides foi mais prevalente entre crianças que moravam em domicílios que possuíam água encanada, e o contrário foi verificado para os ancilostomídeos. Maior prevalência de crianças com ancilostomídeos foi encontrada na zona rural (Tabela 3).

$\mathrm{Na}$ análise bivariada, verificou-se que as variáveis sexo e idade só se mostraram associados a ancilostomídeos e A. lumbricoides, respectivamente. $\mathrm{O}$ primeiro desses parasitos e as geo-helmintíases (global) também se associaram à zona de residência. Por sua vez, escolaridade materna e renda familiar apresentaram-se estatisticamente associados às geo-helmintíases estudadas, a exceção do T. trichiura, que não apresentou asso-

Número e percentual de crianças de 5 a 14 anos incluídas no inquérito sobre geo-helmintíases segundo características demográficas, sócio-econômicas, ambientais e zona de residência. Brasil * 2005.

\begin{tabular}{|c|c|c|c|c|c|c|c|}
\hline \multirow[t]{3}{*}{ Características } & \multicolumn{5}{|c|}{ Zona de residência } & \multicolumn{2}{|c|}{ Total } \\
\hline & \multicolumn{2}{|c|}{ Urbana } & \multicolumn{3}{|c|}{ Rural } & \multirow[b]{2}{*}{$\mathrm{n}$} & \multirow[b]{2}{*}{$\%$} \\
\hline & $\mathrm{n}$ & $\%$ & $\mathbf{n}$ & $\%$ & $\begin{array}{l}\text { Valor } \\
\text { de p }\end{array}$ & & \\
\hline \multicolumn{8}{|l|}{ Sexo } \\
\hline Masculino & 869 & 50,9 & 443 & 54,2 & & 1.211 & 48,0 \\
\hline Feminino & 837 & 49,1 & 374 & 45,8 & 0,12 & 1.312 & 52,0 \\
\hline \multicolumn{8}{|l|}{ Idade (em anos) } \\
\hline $10-14$ & 1.026 & 60,1 & 510 & 62,4 & & 1.536 & 60,9 \\
\hline $5-9$ & 680 & 39,9 & 307 & 37,6 & 0,27 & 987 & 39,1 \\
\hline \multicolumn{8}{|c|}{ Escolaridade da mãe (anos) } \\
\hline$>3$ & 1.134 & 66,5 & 416 & 50,9 & & 1.546 & 61,4 \\
\hline$\leq 3$ & 572 & 33,5 & 401 & 49,1 & 0,00 & 973 & 38,6 \\
\hline \multicolumn{8}{|c|}{ Renda da família (salários mínimos) } \\
\hline$>1$ & 509 & 29,8 & 102 & 12,5 & & 611 & 25,1 \\
\hline$\leq 1$ & 1.123 & 65,8 & 698 & 85,4 & 0,00 & 1.821 & 74,9 \\
\hline \multicolumn{8}{|c|}{ Lixo próximo de casa } \\
\hline Não & 1.037 & 60,9 & 134 & 16,4 & & 1.171 & 46,5 \\
\hline Sim & 667 & 39,1 & 681 & 83,6 & 0,00 & 1.348 & 53,5 \\
\hline \multicolumn{8}{|l|}{ Água encanada } \\
\hline Sim & 396 & 23,2 & 149 & 18,2 & & 545 & 21,6 \\
\hline Não & 1.310 & 76,8 & 668 & 81,8 & 0,00 & 1.978 & 78,4 \\
\hline \multicolumn{8}{|c|}{ Número de pessoas na casa } \\
\hline$\leq 5$ & 953 & 55,9 & 385 & 47,2 & & 1.338 & 53,1 \\
\hline$>5$ & 751 & 44,1 & 430 & 52,8 & 0,00 & 1.181 & 46,9 \\
\hline \multicolumn{8}{|c|}{ Número de cômodos na casa } \\
\hline$>4$ & 670 & 39,4 & 350 & 42,9 & & 1.020 & 40,5 \\
\hline$\leq 4$ & 1.031 & 60,6 & 465 & 57,1 & 0,09 & 1.496 & 59,5 \\
\hline
\end{tabular}

* Refere-se a dez municípios das regiões Norte e Nordeste, com IDH < 0,500 em 1991. 


\begin{tabular}{|c|c|c|c|c|c|}
\hline \multirow[t]{2}{*}{ Variáveis sócio-econômicas } & \multirow{2}{*}{$\begin{array}{l}\text { Crianças } \\
\text { examinadas }\end{array}$} & \multicolumn{4}{|c|}{ Freqüência global } \\
\hline & & $\%$ & $\mathrm{RP}$ & Valor de $\mathrm{p}$ & IC95\% \\
\hline \multicolumn{6}{|l|}{ Zona de residência } \\
\hline Urbana & 1.706 & 32,2 & 1,42 & 0,00 & $1,28-1,57$ \\
\hline Rural & 817 & 45,7 & & & \\
\hline \multicolumn{6}{|l|}{ Sexo } \\
\hline Feminino & 1.211 & 34,7 & 1,10 & 0,06 & $0,99-1,22$ \\
\hline Masculino & 1.312 & 38,3 & & & \\
\hline \multicolumn{6}{|l|}{ Idade (anos) } \\
\hline 10-14 & 1.536 & 36,8 & 0,98 & 0,76 & $0,88-1,09$ \\
\hline $5-9$ & 987 & 36,2 & & & \\
\hline \multicolumn{6}{|l|}{ Escolaridade da mãe (anos) } \\
\hline$>3$ & 1.550 & 30,1 & 1,56 & 0,00 & $1,41-1,72$ \\
\hline$\leq 3$ & 973 & 46,9 & & & \\
\hline \multicolumn{6}{|c|}{ Renda da família (salários mínimos) } \\
\hline$>1$ & 611 & 22,3 & 1,86 & 0,00 & $1,59-2,18$ \\
\hline$\leq 1$ & 1.821 & 41,4 & & & \\
\hline \multicolumn{6}{|l|}{ Lixo próximo de casa } \\
\hline Não & 1.171 & 23,4 & 2,05 & 0,00 & $1,82-2,31$ \\
\hline $\operatorname{Sim}$ & 1.348 & 48,0 & & & \\
\hline \multicolumn{6}{|l|}{ Água encanada } \\
\hline $\operatorname{Sim}$ & 545 & 36,1 & 1,01 & 0,83 & $0,89-1,15$ \\
\hline Não & 1.978 & 36,7 & & & \\
\hline \multicolumn{6}{|l|}{ Número de pessoas na casa } \\
\hline$\leq 5$ & 1.338 & 29,3 & 1,53 & 0,00 & $1,38-1,70$ \\
\hline$>5$ & 1.181 & 44,8 & & & \\
\hline \multicolumn{6}{|l|}{ Número de cômodos na casa } \\
\hline$>4$ & 1.020 & 30,0 & 1,37 & 0,00 & $1,22-1,53$ \\
\hline$\leq 4$ & 1.496 & 41,0 & & & \\
\hline
\end{tabular}

* Refere-se a dez municípios das regiões Norte e Nordeste, com IDH < 0,500 em 1991.

ciação significante com renda familiar. Presença de lixo, número de pessoas e número de cômodos no domicílio também se mostraram associados com cada um dos geohelmintos analisados bem como com o conjunto deles, enquanto a variável água encanada só se mostrou associada a ancilostomídeos e a T. trichiura.

Ao se proceder à análise multivariada (Tabela 4), verificou-se que renda familiar $\leq 1$ salário mínimo ( $\mathrm{OR}=1,75 ; 1,38-2,23)$, escolaridade materna $\leq 3$ anos de estudo (OR = 1,69; 1,39-2,06), presença de lixo próximo ao domicílio $(\mathrm{OR}=$ $1,50 ; 1,22-1,84)$ e mais de 5 pessoas no domicílio $(\mathrm{OR}=1,41 ; 1,17-1,71)$ apresentaram-se estatisticamente associados às infecções pelo conjunto dos três helmintos estudados. Infecções por A. lumbricoides mostraram-se associadas à renda familiar baixa $(\mathrm{OR}=1,78 ; 1,35-2,36)$, mais de 5 pessoas no domicílio ( $\mathrm{OR}=1,55 ; 1,26$ $1,91)$, escolaridade materna $\leq 3$ anos de estudo $(\mathrm{OR}=1,43 ; 1,16-1,76)$, presença de lixo próximo ao domicílio $(\mathrm{OR}=1,27 ; 1,01-1,60)$ e número de cômodos $\leq 4(\mathrm{OR}=1,27$; 1,02-1,58). Com relação aos ancilostomídeos, observou-se associação positiva com residência em zona rural $(\mathrm{OR}=1,97 ; 1,51-2,56)$, presença de lixo próximo ao domicílio $(\mathrm{OR}=1,89 ; 1,38-2,59)$, renda familiar baixa $(\mathrm{OR}=1,74 ; 1,21-2,51)$, escolaridade materna $\leq 3$ anos de estudo $(\mathrm{OR}=1,52$; 1,18-1,96), sexo masculino ( $\mathrm{OR}=1,47 ; 1,15-1$, 88), mais de 5 pessoas no domicílio $(\mathrm{OR}=1,45$; 1,12-1,88) e associação inversa com a idade (OR $=0,78 ; 0,61-0,99)$. Já para a infecção por T. trichiura, os fatores associados foram ausência de água encanada em casa $(\mathrm{OR}=1,79 ; 1,25$ $2,54)$, número de cômodos $\leq 4(\mathrm{OR}=1,56$; 1,15- 
Número de crianças * examinadas, proporção (\%) de geo-helmintíases por espécie e razão de proporção (RP) segundo características demográficas, sócioeconômicas e ambientais. Brasil **, 2005.

\begin{tabular}{|c|c|c|c|c|c|c|c|c|c|c|c|c|c|}
\hline \multirow[t]{2}{*}{ Variáveis } & \multirow{2}{*}{$\begin{array}{c}\text { Crianças } \\
\text { examinadas }\end{array}$} & \multicolumn{4}{|c|}{ Ascaris lumbricoides } & \multicolumn{4}{|c|}{ Ancilostomídeos } & \multicolumn{4}{|c|}{ Trichuris trichiura } \\
\hline & & $\%$ & $\begin{array}{l}\text { Valor } \\
\text { de p }\end{array}$ & RP & IC95\% & $\%$ & $\begin{array}{l}\text { Valor } \\
\text { de p }\end{array}$ & RP & IC95\% & $\%$ & $\begin{array}{l}\text { Valor } \\
\text { de p }\end{array}$ & RP & IC95\% \\
\hline \multicolumn{14}{|c|}{ Zona de residência } \\
\hline Urbana & 1.706 & 24,1 & & & & 10,2 & & & & 12,2 & & & \\
\hline Rural & 817 & 27,2 & 0,10 & 1,13 & $0,98-1,30$ & 25,8 & 0,00 & 2,53 & $2,11-3,04$ & 12,0 & 0,85 & 0,98 & $0,78-1,22$ \\
\hline \multicolumn{14}{|l|}{ Sexo } \\
\hline Feminino & 1.211 & 25,4 & & & & 12,7 & & & & 12,0 & & & \\
\hline Masculino & 1.312 & 24,8 & 0,77 & 0,98 & $0,86-1,12$ & 17,6 & 0,00 & 1,38 & $1,15-1,67$ & 12,3 & 0,77 & 1,03 & $0,84-1,27$ \\
\hline \multicolumn{14}{|l|}{$\begin{array}{l}\text { Idade } \\
\text { (anos) }\end{array}$} \\
\hline $10-14$ & 1.536 & 26,6 & & & & 15,0 & & & & 12,1 & & & \\
\hline $5-9$ & 987 & 22,7 & 0,03 & 0,85 & $0,74-0,98$ & 15,7 & 0,62 & 1,05 & $0,87-1,26$ & 12,3 & 0,91 & 1,01 & $0,82-1,25$ \\
\hline \multicolumn{14}{|l|}{$\begin{array}{l}\text { Escolaridade } \\
\text { da mãe (anos) }\end{array}$} \\
\hline$>3$ & 1.550 & 20,6 & & & & 11,1 & & & & 10,1 & & & \\
\hline$\leq 3$ & 973 & 32,3 & 0,00 & 1,57 & $1,37-1,79$ & 21,9 & 0,00 & 1,97 & $1,64-2,37$ & 15,4 & 0,00 & 1,52 & $1,23-1,87$ \\
\hline \multicolumn{14}{|c|}{$\begin{array}{l}\text { Renda da família } \\
\text { (salários mínimos) }\end{array}$} \\
\hline$>1$ & 611 & 13,7 & & & & 7,0 & & & & 7,7 & & & \\
\hline$\leq 1$ & 1.821 & 29,0 & 0,00 & 2,11 & $1,71-2,60$ & 17,6 & 0,00 & 2,5 & $1,84-3,39$ & 13,6 & 0,00 & 1,76 & $1,31-2,37$ \\
\hline \multicolumn{14}{|l|}{$\begin{array}{l}\text { Lixo próximo } \\
\text { de casa }\end{array}$} \\
\hline Não & 1.171 & 16,3 & & & & 6,8 & & & & 6,8 & & & \\
\hline Sim & 1.348 & 32,7 & 0,00 & 2,01 & $1,72-2,33$ & 22,5 & 0,00 & 3,3 & $2,61-4,17$ & 16,8 & 0,00 & 2,45 & $1,92-3,13$ \\
\hline \multicolumn{14}{|c|}{ Água encanada } \\
\hline Sim & 545 & 29,2 & & & & 11,2 & & & & 10,1 & & & \\
\hline Não & 1.978 & 24,0 & 0,01 & 0,82 & $0,70-0,96$ & 16,4 & 0,00 & 1,46 & $1,13-1,89$ & 12,8 & 0,09 & 1,26 & $0,96-1,66$ \\
\hline \multicolumn{14}{|c|}{$\begin{array}{l}\text { Número de } \\
\text { pessoas na casa }\end{array}$} \\
\hline$\leq 5$ & 1.338 & 19,8 & & & & 10,5 & & & & 8,8 & & & \\
\hline$>5$ & 1.181 & 31,1 & 0,00 & 1,57 & $1,37-1,80$ & 20,6 & 0,00 & 1,95 & $1,61-2,36$ & 15,9 & 0,00 & 1,81 & $1,45-2,24$ \\
\hline \multicolumn{14}{|c|}{$\begin{array}{l}\text { Número de } \\
\text { cômodos na casa }\end{array}$} \\
\hline$>4$ & 1.020 & 20,2 & & & & 10,8 & & & & 9,5 & & & \\
\hline$\leq 4$ & 1.496 & 29,0 & 0,00 & 1,41 & $1,22-1,63$ & 18,0 & 0,00 & 1,69 & $1,38-2,08$ & 14,0 & 0,00 & 1,47 & $1,17-1,84$ \\
\hline
\end{tabular}

* De 5 a 14 anos de idade;

** Refere-se a dez municípios das regiões Norte e Nordeste, com IDH < 0,500 em 1991.

2,12), mais de 5 pessoas no domicílio (OR = 1,39; $1,04-1,90)$, residir em zona urbana $(\mathrm{OR}=0,61$; $0,44-0,84)$ e escolaridade materna $\leq 3$ anos de estudo $(\mathrm{OR}=1,38 ; 1,03-1,84)$.

\section{Discussão}

A elevada freqüência (36,5\%) de crianças infectadas por, pelo menos, uma espécie de geohelminto observada neste estudo confirma que, nos municípios mais pobres do Brasil, as geo-helmintíases ainda representam relevante problema de saúde. Embora a prevalência de crianças com geohelmintíases, na maioria dos municípios analisados, superem aquele observado (18,9\%) entre crianças residentes na zona rural de Uberlândia, Minas Gerais, no Sudeste do país 21, em outros é inferior aos $42 \%$ encontrados por Tsuyuoka et al. 22 em Aracaju, capital do Estado de Sergipe, situada no Nordeste. É possível que questões metodológicas, aliadas a diferenças nas condições sócio-econômicas e no grau de desenvolvimento dos sistemas de saúde locais existentes entre as 
regiões onde se situam os municípios de residência das crianças de cada estudo, possam explicar a ampla variabilidade dos resultados observados no Brasil.

É evidente que a magnitude das geo-helmintíases, nos dias atuais, é inferior aos $86,4 \%$ encontrados por Pellon \& Teixeira 23, na década de 1940, quando as condições de vida das populações do interior do Nordeste eram mais precárias e ainda não se dispunha de terapêutica segura e eficaz para utilização em massa. Entretanto, com a descentralização das ações de saúde que vem possibilitando maior acesso à antihelmínticos de largo espectro e à relativa melhoria das condições de vida da população nas últimas décadas, poder-se-ia esperar que os níveis de prevalência dessas parasitoses fossem mais baixos que os observados.

Chama também a atenção, no presente inquérito, os níveis de prevalência de infecções por A. lumbricoides (25,1\%) e T. trichiura (12,2\%) inferiores àqueles encontrados em Salvador (33,1\% e 25,5\%), em 2003/2004, mesmo após este município ter sido alvo de um programa de saneamento ambiental 24 . A diversidade das condições ecológicas dos municípios estudados, especificamente a umidade do solo é uma das hipóteses que poderia explicar este fato, uma vez que a maioria das crianças incluídas no nosso estudo residia em regiões semi-áridas do Nordeste. Nestas áreas predomina o clima quente e seco que dificulta a disseminação de helmintos 25 , enquanto o contrário ocorre na cidade de Salvador que, por estar situada em região litorânea, apresenta umidade do solo maior em função da quantidade e regularidade das chuvas.

Como referido anteriormente, reconhece-se que está havendo uma redução na prevalência de enteroparasitoses no Brasil, tanto em face dos avanços tecnológicos quanto no campo da saúde como no que se refere ao saneamento ambiental. Contudo, tendo em vista que o referido indicador pode revelar o grau de contaminação ambiental 26 de uma área, a manutenção de elevadas freqüências dessas infecções na periferia das grandes cidades e em zonas rurais, evidencia a necessidade de maior investimento de recursos nestas áreas. Ainda mais que os mesmos são de grande importância, não só para reduzir as parasitoses intestinais, como também outras doenças de maior gravidade tais como diarréia infantil aguda, hepatites virais, dengue, leptospirose, dentre outras. Fortalecem a afirmação anterior, as freqüências mais elevadas de geohelmintos entre crianças que residiam em zona rural, onde a deficiência de saneamento ainda é maior e as condições de vida mais precárias, conforme constatado neste estudo e pelos indicadores di- vulgados pelo Instituto Brasileiro de Geografia e Estatística (IBGE; http://www.sidra.Ibge.gov.br/ bda/acervo/acervo4.asp? $\mathrm{z}=\mathrm{t} \& \mathrm{o}=3$, acessado em 22/Ago/2007).

A exemplo do que tem sido demonstrado na maioria das investigações realizadas no Brasil 27,28, também em nosso estudo as infecções por A. lumbricoides foram as mais prevalentes. Possivelmente, este fato deve-se à própria biologia deste parasita que tem ovos muito resistentes com grande capacidade de aderência a superfícies, fato que representa um fator importante na sua transmissão, uma vez que, presente no ambiente e em alimentos, tais ovos não são removidos com facilidade por lavagens e podem permanecer por até dez anos 29.

Por outro lado, seria de se esperar que a prevalência de T. trichiura fosse maior que a encontrada, conforme demonstrado em outras investigações 30,31 dado que este helminto compartilha com a espécie $A$. lumbricoides o mesmo mecanismo de transmissão e a mesma distribuição geográfica. Talvez, a maior resistência dos ovos de A. lumbricoides, mesmo sob condições adversas do meio, mantenha sua evolução natural, enquanto os de T. trichiura se desenvolvam em menor proporção.

Como observado em outros inquéritos realizados no país, quer atuais 21,32 quer menos recentes 13,33, no presente estudo os ancilostomídeos igualmente foram os geohelmintos mais prevalentes na zona rural. $\mathrm{O}$ achado sugere que as deficiências relativas ao saneamento e à atenção à saúde naquelas áreas têm se modificado aquém do desejado. A carência repercute negativamente na prevenção da ancilostomíase, o que é preocupante, pois a característica fundamental deste parasitismo é a permanente espoliação de sangue dos indivíduos que leva a anemia ferropriva contribuindo para agravar as deficiências nutricionais destas populações.

Apesar de a população deste estudo ser relativamente homogênea no tocante aos indicadores sócio-econômicos, ainda assim foi possível detectar forte associação da renda familiar, escolaridade materna, número de pessoas no domicílio e presença de lixo próximo à residência da criança com a ocorrência tanto pelas três geo-helmintíases em conjunto, como especificamente com pelos A. lumbricoides e ancilostomídeos. Juntamente com o fato da manutenção da associação entre residência em zona rural e ocorrência da ancilostomíase, estes resultados evidenciam, mais uma vez, a relevância das condições sócio-econômicas e ambientais na determinação destas infecções.

Uma limitação na interpretação dos resultados deste estudo refere-se à não-disponibilidade 
da informação sobre o destino dos dejetos. Todavia, considerando que na zona urbana dos municípios investigados a proporção de domicílios ligados à rede de esgotamento sanitária ou que possuíam fossa séptica variava de 0,34\% (Santa Helena) a $32,2 \%$ (Bragança) e, que na zona rural a variação era de 0,06\% em Mucambo a 3,15\% em Ribeiro Gonçalves (IBGE; http://www.sidra. Ibge.gov.br/bda/acervo/acervo4.asp? $\mathrm{z}=\mathrm{t} \& \mathrm{o}=3$, acessado em 22/Ago/2007), é possível supor que o destino inadequado dos dejetos também representa um importante fator na determinação da ocorrência das geo-helmintíases nessas áreas.

\section{Resumo}

Para determinar a prevalência de geo-helmintíases e identificar fatores associados a sua ocorrência, foram realizados inquéritos coprológicos em amostra de crianças entre 5 e 14 anos de idade, residentes em dez municípios brasileiros com baixo Índice de Desenvolvimento Humano. Aplicou-se questionário aos responsáveis, obtendo-se informações sócioeconômicas e ambientais e foi feita coleta de fezes. Estimaram-se prevalências de geohelmintos segundo variáveis de interesse e se avaliaram os fatores de risco mediante regressão logística multinível. Das 2.523 crianças estudadas, $36,5 \%$ eram portadoras de um ou mais geohelmintos (Ascaris lumbricoides 25,1\%; ancilostomídeos $15,3 \%$, Trichuris trichiura 12,2\%). A proporção de geo-helmintíases para o conjunto na zona rural foi 45,7\%; na urbana, 32,2\%. Baixa renda familiar $(O R=1,75 ; 1,38-2,23)$, baixa escolaridade materna $(O R=1,69 ; 1,39-2,06)$, presença de lixo próximo ao domicílio $(O R=1,50 ; 1,22-1,84)$ e maior número de pessoas no domicílio $(O R=1,41 ; 1,17-1,71)$ mostraram-se associadas a tais infecções. Conclui-se que a ocorrência destas parasitoses está relacionada às condições sócio-econômicas e evidencia a importância de intervenções públicas direcionadas à melhoria das condições de vida para sua prevenção.
Vale ainda ressaltar que o tempo decorrido entre a coleta e a leitura das lâminas pode ter ocasionado a redução da sensibilidade do exame, uma vez que para a identificação de ancilostomídeos a observação deve ser feita imediatamente após a preparação das lâminas, fato que significa que a prevalência de geohelmintos encontrada pode estar subestimada. Por conseguinte, são necessários investimentos voltados tanto à melhoria da situação ambiental como às condições sócio-econômicas da população. Ou seja, ações setoriais ao lado de intervenções estruturais direcionadas aos fatores de risco de ocorrência dessas infecções precisam ser adotadas para que, de fato, ocorra e se mantenha uma real melhoria do perfil epidemiológico dessas doenças.

\section{Colaboradores}

E. O. L. Fonseca contribuiu na concepção e desenho do estudo, análise e interpretação dos dados, redação do artigo, aprovação final da versão a ser submetida para publicação. M. C. N. Costa contribuiu na concepção e desenho do estudo, da análise e interpretação dos dados, redação e revisão crítica do artigo, aprovação final da versão a ser submetida para publicação. M. G. Teixeira contribuiu na concepção e desenho do estudo, participou da análise e interpretação dos dados, redação do artigo, aprovação final da versão a ser submetida para publicação. M. L. Barreto contribuiu na concepção e desenho do estudo, da análise e interpretação dos dados, revisão crítica relevante do conteúdo do artigo, aprovação final da versão a ser submetida para publicação. E. H. Carmo contribuiu na concepção e desenho do estudo, análise e interpretação dos dados, revisão crítica relevante do conteúdo do artigo e aprovação final da versão a ser submetida para publicação. 


\section{Referências}

1. Hoste H. Adaptative physiological processes in the host during gastrointestinal parasitism. Int J Parasitol 2001; 31:231-44.

2. Devera R, Cermeño JR, Blanco Y, Morales MCB, Guerra X, Souza M, et al. Prevalencia de blastocistosis y otras parasitosis intestinales en una comunidad rural del Estado Anzoátegui, Venezuela. Parasitol Latinoam 2003; 58:95-100.

3. Matos SMA. Prevalência de enteroparasitoses e sua relação com o estado antropométrico na infância, Salvador-BA [Dissertação de Mestrado]. Salvador: Instituto de Saúde Coletiva, Universidade Federal da Bahia; 2006.

4. Castiñeiras TMPP, Martins FSV. Infecções por helmintos e enteroprotozoários. Rio de Janeiro: Centro de Informações em Saúde para Viajantes, Universidade Federal do Rio de Janeiro; 2000/2002.

5. Chan MS. The global burden of intestinal nematode infections-fifty years on. Parasitol Today 1997; 13:438-43

6. World Health Organization. Deworming for health and development. Report of the Third Global Meeting of the Partners for Parasite Control. Geneva: World Health Organization; 2005.

7. Ehrenberg JP. Por um continente livre de verminoses! Washington DC: Organização Pan-Americana da Saúde; 2002. (Boletim da Organização PanAmericana da Saúde).

8. Zonta ML, Navone GT, Oyhenart EE. Parasitosis intestinales en niños de edad preescolar y escolar: situación actual en poblaciones urbanas, periurbanas y rurales en Brandsen, Buenos Aires, Argentina. Parasitol Latinoam 2007; 62:54-60.

9. Giraldo-Gómez JM, Lora F, Henao LH, Mejía S, Gómez-Marín JE. Prevalencia de giardiasis y parasitos intestinales en preescolares de hogares atendidos en un programa estatal en Armenia, Colombia. Rev Salud Pública 2005; 7:327-38.

10. Carvalho-Costa FA, Gonçalves AQ, Lassance SL, Silva Neto LM, Salmazo CAA, Bóia MN. Giardia lamblia and other intestinal parasitic infections and their relationships with nutritional status in children in Brazilian Amazon. Rev Inst Med Trop São Paulo 2007; 49:147-53.

11. Ferreira GR, Andrade CFS. Alguns aspectos socioeconômicos relacionados a parasitoses intestinais e avaliação de uma intervenção educativa em escolares de Estiva Gerbi, SP. Rev Soc Bras Med Trop 2005; 38:402-5.

12. Coura-Filho P. Distribuição da esquistossomose no espaço urbano. 1. O caso da região metropolitana de Belo Horizonte, Minas Gerais, Brasil. Cad Saúde Pública 1997; 13:415-24.

13. De Carli GA, Mentz M, Rott MB, Silva ACA, Wendorff A, Tasca T, et al. Prevalência das enteroparasitoses na população urbana e rural da região carbonífera da cidade de Arroio dos Ratos, no Estado do Rio Grande do Sul. Rev Bras Farm 1997; 78:83-5.

14. Uchôa CMA, Lobo AGB, Bastos OMP, Matos AD. Parasitoses intestinais: prevalência em creches comunitárias da cidade de Niterói, Rio de Janeiro Brasil. Rev Inst Adolfo Lutz 2001; 60:97-101.
15. Carmo EA, Perez EP, Gerolomo M, Silva MP, Alves RMS. Plano nacional de vigilância e controle das enteroparasitoses. http://portal.saude.gov.br/por tal/arquivos/pdf/Enteroparasitoses_Pano_nacio nal_\%2006\%2007\%202005.pdf (acessado em 13/ $\mathrm{Jul} / 2007$ )

16. Ministério da Saúde/Organização Pan-Americana da Saúde. Avaliação de impacto na saúde das ações de saneamento: marco conceitual e estratégia metodológica. Brasília: Organização Pan-Americana da Saúde/Ministério da Saúde; 2004.

17. Fundação Nacional de Saúde. Portaria nº 176. Aprova os critérios e procedimentos para aplicação de recursos financeiros. Diário Oficial da União 2000; 28 mar.

18. Katz N, Chaves A, Pellegrino J. A simple device for quantitative stool thick-smear technique in schistosomiasis mansoni. Rev Inst Med Trop São Paulo 1972; 14:397-400.

19. Hoffmann WA, Pons JA, Janer JL. The sedimentation concentration method in schistosomiasis mansoni. PR J Public Health Trop Med 1934; 9: 283-91.

20. Davis CS. Statistical methods for the analysis of repeated measurements. New York: Springer; 2002.

21. Ribeiro MCM, Marçal Júnior O. Prevalência e fatores de risco para geo-helmintíases em escolares da zona rural de Uberlândia (MG). Rev Patol Trop 2003; 32:105-15.

22. Tsuyuoka R, Bailey JW, Guimarães AMD'AN, Gurgel RQ, Cuevas LE. Anemia and intestinal parasitic infections in primary school students in Aracaju, Sergipe, Brazil. Cad Saúde Pública 1999; 15: 413-21.

23. Pellon AB, Teixeira I. Distribuição da esquistossomose mansônica no Brasil. Rio de Janeiro: Divisão da Organização Sanitária; 1950.

24. Barreto ML. Avaliação do impacto epidemiológico do Programa de Saneamento Ambiental da Baía de Todos os Santos (Bahia Azul). Salvador: Instituto de Saúde Coletiva, Universidade Federal da Bahia; 2006.

25. Camillo-Coura L. Contribuição ao estudo das geohelmintíases [Tese de Doutorado]. Rio de Janeiro: Faculdade de Medicina, Universidade Federal do Rio de Janeiro; 1970.

26. Carneiro FF, Cifuentes E, Tellez-Rojo MM, Romieu I. The risk of Ascaris lumbricoides infection in children as an environmental health indicator to guide preventive activities in Caparao and Alto Caparao, Brazil. Bull World Health Organ 2002; 80:40-6.

27. Pereira CW, Santos FN. Prevalência de geo-helmintíases em crianças atendidas na rede pública de saúde de Neópolis, município do estado de Sergipe. Rev Bras Anál Clín 2005; 37:111-4.

28. Ferreira P, Lima MR, Oliveira FB, Pereira MLM, Ramos LBM, Marçal MG, et al. Ocorrência de parasitos e comensais intestinais em crianças de escola localizada em assentamento de sem-terras em Campo Florido, Minas Gerais, Brasil. Rev Soc Bras Med Trop 2003; 36:109-11. 
29. Luduvice M. Experiência da Companhia de Saneamento do Distrito Federal na reciclagem agrícola de biossólido. In: BettiolW, Camargo OA, organizadores. Impacto ambiental do uso agrícola do lodo de esgoto. Jaguariúna: Embrapa Meio Ambiente; 2000. p. 245-58.

30. Bezerra FSM, Oliveira MF, Miranda ALL, Pinheiro MCC, Teles RMA. Incidência de parasitos intestinais em material sub-ungueal e fecal em crianças da Creche Aprisco - Fortaleza, CE. Rev Bras Anál Clín 2003; 35:39-40.

31. Costa-Macedo LM, Machado-Silva JR, RodriguesSilva R, Oliveira LM, Vianna MSR. Enteroparasitoses em pré-escolares de comunidades favelizadas da Cidade do Rio de Janeiro, Brasil. Cad Saúde Pública 1998; 14:851-5.
32. Mascarini LL, Donalísio-Cordeiro MR. Helmintíases em crianças institucionalizadas em creches no município de Botucatu/SP, Brasil. Rev Patol Trop 2007; 36:149-58.

33. Barbosa FS, Pessoa D, Pinto RF, Barbosa JM, Rodrigues BA. Levantamento seccionais sobre a esquistossomose no Nordeste do Brasil. III. Estado de Pernambuco (município de São Lourenço da Mata). Rev Soc Bras Med Trop 1970; IV:269-80.

Recebido em 26/Jun/2008

Versão final reapresentada em 16/Jun/2009

Aprovado em 14/Out/2009 